\title{
Hybrid methods for common solutions in Hilbert spaces with applications
}

Lijuan Sun*

Correspondence: kfsunlj@yeah.net Kaifeng Vocational College of

Culture and Arts, Kaifeng, Henan, China

\begin{abstract}
In this paper, hybrid methods are investigated for treating common solutions of nonlinear problems. A strong convergence theorem is established in the framework of real Hilbert spaces.
\end{abstract}

Keywords: equilibrium problem; fixed point; projection; variational inequality; zero point

\section{Introduction and preliminaries}

Common solutions to variational inclusion, equilibrium and fixed point problems have been recently extensively investigated based on iterative methods; see [1-33] and the references therein. The motivation for this subject is mainly to its possible applications to mathematical modeling of concrete complex problems, which use more than one constraint. The aim of this paper is to investigate a common solution of variational inclusion, equilibrium and fixed point problems. The organization of this paper is as follows. In Section 1, we provide some necessary preliminaries. In Section 2, a hybrid method is introduced and analyzed. Strong convergence theorems are established in the framework of Hilbert spaces. In Section 3, applications of the main results are discussed.

In what follows, we always assume that $H$ is a real Hilbert space with the inner product $\langle\cdot, \cdot\rangle$ and the norm $\|\cdot\|$. Let $C$ be a nonempty, closed, and convex subset of $H$ and let $P_{C}$ be the metric projection from $H$ onto $C$. Let $S: C \rightarrow C$ be a mapping. $F(S)$ stands for the fixed point set of $S$; that is, $F(S):=\{x \in C: x=S x\}$.

Recall that $S$ is said to be contractive iff there exists a constant $\alpha \in[0,1)$ such that

$$
\|S x-S y\| \leq \alpha\|x-y\|, \quad \forall x, y \in C .
$$

If $\alpha=1$, then $S$ is said to be nonexpansive. Let $A: C \rightarrow H$ be a mapping. If $C$ is nonempty closed and convex, then the fixed point set of $S$ is nonempty.

Recall that $A$ is said to be monotone iff

$$
\langle A x-A y, x-y\rangle \geq 0, \quad \forall x, y \in C .
$$

Recall that $A$ is said to be strongly monotone iff there exists a constant $\alpha>0$ such that

$$
\langle A x-A y, x-y\rangle \geq \alpha\|x-y\|^{2}, \quad \forall x, y \in C
$$

(O\#CPRSun; licensee Springer. This is an Open Access article distributed under the terms of the Creative Commons Attribution License (http://creativecommons.org/licenses/by/2.0), which permits unrestricted use, distribution, and reproduction in any medium, provided the original work is properly cited. 
For such a case, $A$ is also said to be $\alpha$-strongly monotone. Recall that $A$ is said to be inversestrongly monotone iff there exists a constant $\alpha>0$ such that

$$
\langle A x-A y, x-y\rangle \geq \alpha\|A x-A y\|^{2}, \quad \forall x, y \in C .
$$

For such a case, $A$ is also said to be $\alpha$-inverse-strongly monotone.

Recall that a set-valued mapping $M: H \rightrightarrows H$ is said to be monotone iff, for all $x, y \in H$, $f \in M x$, and $g \in M y$ imply $\langle x-y, f-g\rangle>0$. M is maximal iff the graph $\operatorname{Graph}(M)$ of $R$ is not properly contained in the graph of any other monotone mapping. It is well known that a monotone mapping $M$ is maximal if and only if, for any $(x, f) \in H \times H,\langle x-y, f-g\rangle \geq 0$, for all $(y, g) \in \operatorname{Graph}(M)$ implies $f \in R x$. For a maximal monotone operator $M$ on $H$, and $r>0$, we may define the single-valued resolvent $J_{r}: H \rightarrow D(M)$, where $D(M)$ denote the domain of $M$. It is well known that $J_{r}$ is firmly nonexpansive, and $M^{-1}(0)=F\left(J_{r}\right)$, where $F\left(J_{r}\right):=\left\{x \in D(M): x=J_{r} x\right\}$, and $M^{-1}(0):=\{x \in H: 0 \in M x\}$.

Let $A: C \rightarrow H$ be a inverse-strongly monotone mapping, and let $F$ be a bifunction of $C \times$ $C$ into $\mathbb{R}$, where $\mathbb{R}$ denotes the set of real numbers. We consider the following generalized equilibrium problem.

Find $x \in C$ such that $F(x, y)+\langle A x, y-x\rangle \geq 0, \quad \forall y \in C$.

In this paper, the set of such an $x \in C$ is denoted by $\operatorname{EP}(F, A)$.

To study the equilibrium problems (1.1), we may assume that $F$ satisfies the following conditions:

(A1) $F(x, x)=0$ for all $x \in C$;

(A2) $F$ is monotone, i.e., $F(x, y)+F(y, x) \leq 0$ for all $x, y \in C$;

(A3) for each $x, y, z \in C$,

$$
\limsup _{t \downarrow 0} F(t z+(1-t) x, y) \leq F(x, y)
$$

(A4) for each $x \in C, y \mapsto F(x, y)$ is convex and weakly lower semicontinuous.

In order to prove our main results, we also need the following lemmas.

Lemma 1.1 [34] Assume that $\left\{\alpha_{n}\right\}$ is a sequence of nonnegative real numbers such that

$$
\alpha_{n+1} \leq\left(1-\gamma_{n}\right) \alpha_{n}+\delta_{n}
$$

where $\left\{\gamma_{n}\right\}$ is a sequence in $(0,1)$ and $\left\{\delta_{n}\right\}$ is a sequence such that

(1) $\sum_{n=1}^{\infty} \gamma_{n}=\infty$;

(2) $\lim \sup _{n \rightarrow \infty} \delta_{n} / \gamma_{n} \leq 0$ or $\sum_{n=1}^{\infty}\left|\delta_{n}\right|<\infty$.

Then $\lim _{n \rightarrow \infty} \alpha_{n}=0$.

Lemma 1.2 [35] Let $F: C \times C \rightarrow \mathbb{R}$ be a bifunction satisfying (A1)-(A4). Then, for any $r>0$ and $x \in H$, there exists $z \in C$ such that

$$
F(z, y)+\frac{1}{r}\langle y-z, z-x\rangle \geq 0, \quad \forall y \in C .
$$


Define a mapping $T_{r}: H \rightarrow C$ as follows:

$$
T_{r} x=\left\{z \in C: F(z, y)+\frac{1}{r}\langle y-z, z-x\rangle \geq 0, \forall y \in C\right\}, \quad x \in H
$$

then the following conclusions hold:

(1) $T_{r}$ is single-valued;

(2) $T_{r}$ is firmly nonexpansive, i.e., for any $x, y \in H$,

$$
\left\|T_{r} x-T_{r} y\right\|^{2} \leq\left\langle T_{r} x-T_{r} y, x-y\right\rangle
$$

(3) $F\left(T_{r}\right)=E P(F)$;

(4) $E P(F)$ is closed and convex.

Let $\left\{S_{i}: C \rightarrow C\right\}$ be a family of infinitely nonexpansive mappings and $\left\{\gamma_{i}\right\}$ be a nonnegative real sequence with $0 \leq \gamma_{i}<1, \forall i \geq 1$. For $n \geq 1$ define a mapping $W_{n}: C \rightarrow C$ as follows:

$$
\begin{aligned}
& U_{n, n+1}=I, \\
& U_{n, n}=\gamma_{n} S_{n} U_{n, n+1}+\left(1-\gamma_{n}\right) I, \\
& U_{n, n-1}=\gamma_{n-1} S_{n-1} U_{n, n}+\left(1-\gamma_{n-1}\right) I, \\
& \vdots \\
& U_{n, k}=\gamma_{k} S_{k} U_{n, k+1}+\left(1-\gamma_{k}\right) I, \\
& U_{n, k-1}=\gamma_{k-1} S_{k-1} U_{n, k}+\left(1-\gamma_{k-1}\right) I, \\
& \vdots \\
& U_{n, 2}=\gamma_{2} S_{2} U_{n, 3}+\left(1-\gamma_{2}\right) I, \\
& W_{n}=U_{n, 1}=\gamma_{1} S_{1} U_{n, 2}+\left(1-\gamma_{1}\right) I .
\end{aligned}
$$

Such a mapping $W_{n}$ is nonexpansive from $C$ to $C$ and it is called a $W$-mapping generated by $S_{n}, S_{n-1}, \ldots, S_{1}$ and $\gamma_{n}, \gamma_{n-1}, \ldots, \gamma_{1}$.

Lemma 1.3 [36] Let $\left\{S_{i}: C \rightarrow C\right\}$ be a family of infinitely nonexpansive mappings with a nonempty common fixed point set and let $\left\{\gamma_{i}\right\}$ be a real sequence such that $0<\gamma_{i} \leq l<1$, where $l$ is some real number, $\forall i \geq 1$. Then

(1) $W_{n}$ is nonexpansive and $F\left(W_{n}\right)=\bigcap_{i=1}^{\infty} F\left(S_{i}\right)$, for each $n \geq 1$;

(2) for each $x \in C$ and for each positive integer $k$, the limit $\lim _{n \rightarrow \infty} U_{n, k}$ exists;

(3) the mapping $W: C \rightarrow C$ defined by

$$
W x:=\lim _{n \rightarrow \infty} W_{n} x=\lim _{n \rightarrow \infty} U_{n, 1} x, \quad x \in C,
$$

is a nonexpansive mapping satisfying $F(W)=\bigcap_{i=1}^{\infty} F\left(S_{i}\right)$ and it is called the $W$-mapping generated by $S_{1}, S_{2}, \ldots$ and $\gamma_{1}, \gamma_{2}, \ldots$. 
Lemma 1.4 [27] Let $\left\{S_{i}: C \rightarrow C\right\}$ be a family of infinitely nonexpansive mappings with a nonempty common fixed point set and let $\left\{\gamma_{i}\right\}$ be a real sequence such that $0<\gamma_{i} \leq l<1$, $\forall i \geq 1$. If $K$ is any bounded subset of $C$, then

$$
\lim _{n \rightarrow \infty} \sup _{x \in K}\left\|W x-W_{n} x\right\|=0 .
$$

Throughout this paper, we always assume that $0<\gamma_{i} \leq l<1, \forall i \geq 1$.

Lemma 1.5 [37] Let $B: C \rightarrow H$ be a mapping and let $M: H \rightrightarrows H$ be a maximal monotone operator. Then $F\left(J_{r}(I-s B)\right)=(B+M)^{-1}(0)$.

Lemma 1.6 [38] Let $\left\{x_{n}\right\}$ and $\left\{y_{n}\right\}$ be bounded sequences in $H$ and let $\left\{\beta_{n}\right\}$ be a sequence in $(0,1)$ with $0<\liminf _{n \rightarrow \infty} \beta_{n} \leq \limsup _{n \rightarrow \infty} \beta_{n}<1$. Suppose that $x_{n+1}=\left(1-\beta_{n}\right) y_{n}+\beta_{n} x_{n}$ for all $n \geq 0$ and

$$
\limsup _{n \rightarrow \infty}\left(\left\|y_{n+1}-y_{n}\right\|-\left\|x_{n+1}-x_{n}\right\|\right) \leq 0 .
$$

Then $\lim _{n \rightarrow \infty}\left\|y_{n}-x_{n}\right\|=0$.

Lemma 1.7 [39] Let $A: C \rightarrow H$ a Lipschitz monotone mapping and let $N_{C} x$ be the normal cone to $C$ at $x \in C$; that is, $N_{C} x=\{y \in H:\langle x-u, y\rangle, \forall u \in C\}$. Define

$$
D x= \begin{cases}A x+N_{C} x, & x \in C, \\ \varnothing & x \notin C .\end{cases}
$$

Then $D$ is maximal monotone and $0 \in D x$ if and only if $x \in V I(C, A)$.

\section{Main results}

Theorem 2.1 Let $C$ be a nonempty closed convex subset of a Hilbert space $H$ and $F$ a bifunction from $C \times C$ to $\mathbb{R}$ which satisfies (A1)-(A4). Let $A_{1}: C \rightarrow H$ be a $\delta_{1}$-inversestrongly monotone mapping, $A_{2}: C \rightarrow H$ be a $\delta_{2}$-inverse-strongly monotone mapping, $A_{3}$ : $C \rightarrow H$ be a $\delta_{3}$-inverse-strongly monotone mapping, $M_{1}: H \rightrightarrows H$ a maximal monotone operator such that $\operatorname{Dom}\left(M_{1}\right) \subset C$ and $M_{2}: H \rightrightarrows H$ a maximal monotone operator such that $\operatorname{Dom}\left(M_{2}\right) \subset C$. Let $\left\{S_{i}: C \rightarrow C\right\}$ be a family of infinitely nonexpansive mappings. Assume that $\Omega:=\bigcap_{i=1}^{\infty} F\left(S_{i}\right) \cap E P\left(F, A_{3}\right) \cap\left(A_{1}+M_{1}\right)^{-1}(0) \cap\left(A_{2}+M_{2}\right)^{-1}(0) \neq \emptyset$. Let $x_{1} \in C$ and $\left\{x_{n}\right\}$ be a sequence generated by

$$
\left\{\begin{array}{l}
z_{n}=J_{s_{n}}\left(u_{n}-s_{n} A_{2} u_{n}\right), \\
x_{n+1}=\alpha_{n} u+\beta_{n} x_{n}+\gamma_{n} W_{n} J_{r_{n}}\left(z_{n}-r_{n} A_{1} z_{n}\right), \quad \forall n \geq 1,
\end{array}\right.
$$

where $u$ is a fixed element in $C, u_{n}$ is such that

$$
F\left(u_{n}, y\right)+\left\langle A_{3} x_{n}, y-u_{n}\right\rangle+\frac{1}{\lambda_{n}}\left\langle y-u_{n}, u_{n}-x_{n}\right\rangle \geq 0, \quad \forall y \in C,
$$

$\left\{W_{n}: C \rightarrow C\right\}$ is the sequence generated in (1.2), $\left\{\alpha_{n}\right\},\left\{\beta_{n}\right\}$, and $\left\{\gamma_{n}\right\}$ are sequences in $(0,1)$ such that $\alpha_{n}+\beta_{n}+\gamma_{n}=1$ for each $n \geq 1$ and $\left\{r_{n}\right\},\left\{s_{n}\right\}$, and $\left\{\lambda_{n}\right\}$ are positive number sequences. Assume that the above control sequences satisfy the following restrictions: 
(a) $0<a \leq \lambda_{n} \leq b<2 \delta_{3}, 0<a^{\prime} \leq r_{n} \leq b^{\prime}<2 \delta_{1}, 0<\bar{a} \leq s_{n} \leq \bar{b}<2 \delta_{2}$;

(b) $\lim _{n \rightarrow \infty} \alpha_{n}=0$ and $\sum_{n=1}^{\infty} \alpha_{n}=\infty$;

(c) $0<\liminf _{n \rightarrow \infty} \beta_{n} \leq \lim \sup _{n \rightarrow \infty} \beta_{n}<1$;

(d) $\lim _{n \rightarrow \infty}\left|\lambda_{n}-\lambda_{n+1}\right|=\lim _{n \rightarrow \infty}\left|s_{n}-s_{n+1}\right|=\lim _{n \rightarrow \infty}\left|r_{n}-r_{n+1}\right|=0$.

Then the sequence $\left\{x_{n}\right\}$ converges strongly to $\bar{x} \in \Omega$, where $\bar{x}=P_{\Omega} u$.

Proof First, we show that the mapping $I-r_{n} A_{1}, I-s_{n} A_{2}$, and $I-\lambda_{n} A_{3}$ are nonexpansive. Indeed, we find from the restriction (a) that

$$
\begin{aligned}
\| & \left(I-r_{n} A_{1}\right) x-\left(I-r_{n} A_{1}\right) y \|^{2} \\
& =\|x-y\|^{2}-2 r_{n}\left\langle x-y, A_{1} x-A_{1} y\right\rangle+r_{n}^{2}\left\|A_{1} x-A_{1} y\right\|^{2} \\
& \leq\|x-y\|^{2}-2 r_{n} \delta_{1}\left\|A_{1} x-A_{1} y\right\|^{2}+r_{n}^{2}\left\|A_{1} x-A_{1} y\right\|^{2} \\
& =\|x-y\|^{2}+r_{n}\left(r_{n}-2 \delta_{1}\right)\left\|A_{1} x-A_{1} y\right\|^{2} \\
& \leq\|x-y\|^{2}, \quad \forall x, y \in C,
\end{aligned}
$$

which implies that the mapping $I-r_{n} A_{1}$ is nonexpansive. In the same way, we find $I-s_{n} A_{2}$ and $I-\lambda_{n} A_{3}$ are also nonexpansive. Put $y_{n}=J_{r_{n}}\left(z_{n}-r_{n} A_{1} z_{n}\right)$. Fixing $x^{*} \in \Omega$, we find

$$
\begin{aligned}
\left\|y_{n}-x^{*}\right\| & =\left\|J_{r_{n}}\left(z_{n}-r_{n} A_{1} z_{n}\right)-J_{r_{n}}\left(x^{*}-r_{n} A_{1} x^{*}\right)\right\| \\
& \leq\left\|z_{n}-x^{*}\right\| \\
& =\left\|J_{s_{n}}\left(u_{n}-s_{n} A_{2} u_{n}\right)-J_{s_{n}}\left(x^{*}-s_{n} A_{2} x^{*}\right)\right\| \\
& \leq\left\|T_{\lambda_{n}}\left(I-\lambda_{n} A_{3}\right) x_{n}-T_{\lambda_{n}}\left(I-\lambda_{n} A_{3}\right) x^{*}\right\| \\
& \leq\left\|x_{n}-x^{*}\right\| .
\end{aligned}
$$

It follows that

$$
\begin{aligned}
\left\|x_{n+1}-x^{*}\right\| & =\left\|\alpha_{n} u+\beta_{n} x_{n}+\gamma_{n} W_{n} y_{n}-x^{*}\right\| \\
& \leq \alpha_{n}\left\|u-x^{*}\right\|+\beta_{n}\left\|x_{n}-x^{*}\right\|+\gamma_{n}\left\|W_{n} y_{n}-x^{*}\right\| \\
& \leq \alpha_{n}\left\|u-x^{*}\right\|+\left(1-\alpha_{n}\right)\left\|x_{n}-x^{*}\right\| .
\end{aligned}
$$

This implies that $\left\{x_{n}\right\}$ is bounded, and so are $\left\{y_{n}\right\},\left\{z_{n}\right\}$, and $\left\{u_{n}\right\}$. Without loss of generality, we can assume that there exists a bounded set $K \subset C$ such that $x_{n}, y_{n}, z_{n}, u_{n} \in K$. Notice that

$$
F\left(u_{n+1}, y\right)+\frac{1}{\lambda_{n+1}}\left\langle y-u_{n+1}, u_{n+1}-\left(I-r_{n+1} A_{3}\right) x_{n+1}\right\rangle \geq 0, \quad \forall y \in C,
$$

and

$$
F\left(u_{n}, y\right)+\frac{1}{\lambda_{n}}\left\langle y-u_{n}, u_{n}-\left(I-r_{n} A_{3}\right) x_{n}\right\rangle \geq 0, \quad \forall y \in C .
$$

Let $y=u_{n}$ in (2.1) and $y=u_{n+1}$ in (2.2). By adding these two inequalities, we obtain

$$
\left\langle u_{n+1}-u_{n}, \frac{u_{n}-\left(I-\lambda_{n} A_{3}\right) x_{n}}{\lambda_{n}}-\frac{u_{n+1}-\left(I-\lambda_{n+1} A_{3}\right) x_{n+1}}{\lambda_{n+1}}\right\rangle \geq 0 .
$$


It follows that

$$
\begin{aligned}
\left\|u_{n+1}-u_{n}\right\|^{2} \leq & \left\langle u_{n+1}-u_{n},\left(I-\lambda_{n+1} A_{3}\right) x_{n+1}-\left(I-\lambda_{n} A_{3}\right) x_{n}\right. \\
& \left.+\left(1-\frac{\lambda_{n}}{\lambda_{n+1}}\right)\left(u_{n+1}-\left(I-\lambda_{n+1} A_{3}\right) x_{n+1}\right)\right\rangle \\
\leq & \left\|u_{n+1}-u_{n}\right\|\left(\left\|\left(I-\lambda_{n+1} A_{3}\right) x_{n+1}-\left(I-\lambda_{n} A_{3}\right) x_{n}\right\|\right. \\
& \left.\left.+\left|1-\frac{\lambda_{n}}{\lambda_{n+1}}\right| \| u_{n+1}-\left(I-\lambda_{n+1} A_{3}\right) x_{n+1}\right) \|\right) .
\end{aligned}
$$

It follows that

$$
\begin{aligned}
\left\|u_{n+1}-u_{n}\right\| \leq & \left\|\left(I-\lambda_{n+1} A_{3}\right) x_{n+1}-\left(I-\lambda_{n} A_{3}\right) x_{n}\right\| \\
& +\frac{\left|\lambda_{n+1}-\lambda_{n}\right|}{\lambda_{n+1}}\left\|u_{n+1}-\left(I-\lambda_{n+1} A_{3}\right) x_{n+1}\right\| \\
= & \left\|\left(I-\lambda_{n+1} A_{3}\right) x_{n+1}-\left(I-\lambda_{n+1} A_{3}\right) x_{n}+\left(I-\lambda_{n+1} A_{3}\right) x_{n}-\left(I-\lambda_{n} A_{3}\right) x_{n}\right\| \\
& +\frac{\left|\lambda_{n+1}-\lambda_{n}\right|}{\lambda_{n+1}}\left\|u_{n+1}-\left(I-\lambda_{n+1} A_{3}\right) x_{n+1}\right\| \\
\leq & \left\|x_{n+1}-x_{n}\right\|+\left|\lambda_{n+1}-\lambda_{n}\right| M_{1},
\end{aligned}
$$

where $M_{1}$ is an appropriate constant such that

$$
M_{1}=\sup _{n \geq 1}\left\{\left\|A_{3} x_{n}\right\|+\frac{\left\|u_{n+1}-\left(I-\lambda_{n+1} A_{3}\right) x_{n+1}\right\|}{a}\right\} .
$$

Since $J_{s_{n}}$ is firmly nonexpansive, we find that

$$
\begin{aligned}
& \left\|z_{n+1}-z_{n}\right\| \\
& \quad \leq\left\|u_{n+1}-s_{n+1} A_{2} u_{n+1}-\left(u_{n}-s_{n} A_{2} u_{n}\right)\right\| \\
& \quad=\left\|\left(I-s_{n+1} A_{2}\right) u_{n+1}-\left(I-s_{n+1} A_{2}\right) u_{n}+\left(s_{n}-s_{n+1}\right) A_{2} u_{n}\right\| \\
& \quad \leq\left\|u_{n+1}-u_{n}\right\|+\left|s_{n}-s_{n+1}\right|\left\|A_{2} u_{n}\right\| .
\end{aligned}
$$

Combining (2.3) with (2.4) yields

$$
\left\|z_{n+1}-z_{n}\right\| \leq\left\|x_{n+1}-x_{n}\right\|+\left|\lambda_{n+1}-\lambda_{n}\right| M_{1}+\left|s_{n}-s_{n+1}\right|\left\|A_{2} u_{n}\right\| .
$$

Since $J_{r_{n}}$ is also firmly nonexpansive, we find that

$$
\left\|y_{n+1}-y_{n}\right\| \leq\left\|z_{n+1}-z_{n}\right\|+\left|r_{n}-r_{n+1}\right|\left\|A_{1} z_{n}\right\| \text {. }
$$

Substituting (2.5) into (2.6), we see that

$$
\left\|y_{n+1}-y_{n}\right\| \leq\left\|x_{n+1}-x_{n}\right\|+\left(\left|r_{n+1}-r_{n}\right|+\left|\lambda_{n}-\lambda_{n+1}\right|+\left|s_{n}-s_{n+1}\right|\right) M_{2} \text {, }
$$


where $M_{2}$ is an appropriate constant such that

$$
M_{2}=\max \left\{\sup _{n \geq 1}\left\{\left\|A_{1} z_{n}\right\|\right\}, \sup _{n \geq 1}\left\{\left\|A_{2} u_{n}\right\|\right\}, M_{1}\right\}
$$

Since $W_{n}$ is nonexpansive, we find that

$$
\begin{aligned}
& \left\|W_{n+1} y_{n+1}-W_{n} y_{n}\right\| \\
& \quad=\left\|W_{n+1} y_{n+1}-W y_{n+1}+W y_{n+1}-W y_{n}+W y_{n}-W_{n} y_{n}\right\| \\
& \leq\left\|W_{n+1} y_{n+1}-W y_{n+1}\right\|+\left\|W y_{n+1}-W y_{n}\right\|+\left\|W y_{n}-W_{n} y_{n}\right\| \\
& \quad \leq \sup _{x \in K}\left\{\left\|W_{n+1} x-W x\right\|+\left\|W x-W_{n} x\right\|\right\}+\left\|y_{n+1}-y_{n}\right\|,
\end{aligned}
$$

where $K$ is the bounded subset of $C$ defined as above. Substituting (2.7) into (2.8), we find that

$$
\begin{aligned}
\left\|W_{n+1} y_{n+1}-W_{n} y_{n}\right\| \leq & \sup _{x \in K}\left\{\left\|W_{n+1} x-W x\right\|+\left\|W x-W_{n} x\right\|\right\}+\left\|x_{n+1}-x_{n}\right\| \\
& +\left(\left|r_{n+1}-r_{n}\right|+\left|\lambda_{n}-\lambda_{n+1}\right|+\left|s_{n}-s_{n+1}\right|\right) M_{2} .
\end{aligned}
$$

Letting

$$
x_{n+1}=\left(1-\beta_{n}\right) v_{n}+\beta_{n} x_{n},
$$

we see that

$$
\begin{aligned}
v_{n+1}-v_{n}= & \frac{\alpha_{n+1} u+\gamma_{n+1} W_{n+1} y_{n+1}}{1-\beta_{n+1}}-\frac{\alpha_{n} u+\gamma_{n} W_{n} y_{n}}{1-\beta_{n}} \\
= & \frac{\alpha_{n+1}}{1-\beta_{n+1}} u+\frac{1-\alpha_{n+1}-\beta_{n+1}}{1-\beta_{n+1}} W_{n+1} y_{n+1} \\
& -\left(\frac{\alpha_{n}}{1-\beta_{n}} u+\frac{1-\alpha_{n}-\beta_{n}}{1-\beta_{n}} W_{n} y_{n}\right) \\
= & \frac{\alpha_{n+1}}{1-\beta_{n+1}}\left(u-W_{n+1} y_{n+1}\right)-\frac{\alpha_{n}}{1-\beta_{n}}\left(u-W_{n} y_{n}\right) \\
& +W_{n+1} y_{n+1}-W_{n} y_{n} .
\end{aligned}
$$

Hence, we have

$$
\begin{aligned}
\left\|v_{n+1}-v_{n}\right\| \leq & \frac{\alpha_{n+1}}{1-\beta_{n+1}}\left\|u-W_{n+1} y_{n+1}\right\|+\frac{\alpha_{n}}{1-\beta_{n}}\left\|u-W_{n} y_{n}\right\| \\
& +\left\|W_{n+1} y_{n+1}-W_{n} y_{n}\right\| .
\end{aligned}
$$

Substituting (2.9) into (2.10), we find that

$$
\begin{aligned}
\left\|v_{n+1}-v_{n}\right\|-\left\|x_{n+1}-x_{n}\right\| \leq & \frac{\alpha_{n+1}}{1-\beta_{n+1}}\left\|u-W_{n+1} y_{n+1}\right\|+\frac{\alpha_{n}}{1-\beta_{n}}\left\|u-W_{n} y_{n}\right\| \\
& +\sup _{x \in K}\left\{\left\|W_{n+1} x-W x\right\|+\left\|W x-W_{n} x\right\|\right\} \\
& +\left(\left|r_{n+1}-r_{n}\right|+\left|\lambda_{n}-\lambda_{n+1}\right|+\left|s_{n}-s_{n+1}\right|\right) M_{2} .
\end{aligned}
$$


It follows from Lemma 1.4 that

$$
\limsup _{n \rightarrow \infty}\left(\left\|v_{n+1}-v_{n}\right\|-\left\|x_{n+1}-x_{n}\right\|\right) \leq 0
$$

In view of Lemma 1.6, we find that $\lim _{n \rightarrow \infty}\left\|v_{n}-x_{n}\right\|=0$. It follows that

$$
\lim _{n \rightarrow \infty}\left\|x_{n+1}-x_{n}\right\|=0
$$

For any $x^{*} \in \Omega$, we see that

$$
\left\|x_{n+1}-x^{*}\right\|^{2} \leq \alpha_{n}\left\|u-x^{*}\right\|^{2}+\beta_{n}\left\|x_{n}-x^{*}\right\|^{2}+\gamma_{n}\left\|y_{n}-x^{*}\right\|^{2} \text {. }
$$

Since

$$
\begin{aligned}
\left\|y_{n}-x^{*}\right\|^{2} & \leq\left\|\left(I-r_{n} A_{1}\right) z_{n}-\left(I-r_{n} A_{1}\right) x^{*}\right\|^{2} \\
& =\left\|z_{n}-x^{*}\right\|^{2}-2 r_{n}\left\langle z_{n}-x^{*}, A_{1} z_{n}-A_{1} x^{*}\right\rangle+r_{n}^{2}\left\|A_{1} z_{n}-A_{1} x^{*}\right\|^{2} \\
& \leq\left\|z_{n}-x^{*}\right\|^{2}-2 r_{n} \delta_{1}\left\|A_{1} z_{n}-A_{1} x^{*}\right\|^{2}+r_{n}^{2}\left\|A_{1} z_{n}-A_{1} x^{*}\right\|^{2} \\
& =\left\|x_{n}-x^{*}\right\|^{2}+r_{n}\left(r_{n}-2 \delta_{1}\right)\left\|A_{1} z_{n}-A_{1} x^{*}\right\|^{2}
\end{aligned}
$$

we find that

$$
\begin{aligned}
& \gamma_{n} r_{n}\left(2 \delta_{1}-r_{n}\right)\left\|A_{1} z_{n}-A_{1} x^{*}\right\|^{2} \\
& \quad \leq \alpha_{n}\left\|u-x^{*}\right\|^{2}+\left(\left\|x_{n}-x^{*}\right\|+\left\|x_{n+1}-x^{*}\right\|\right)\left\|x_{n}-x_{n+1}\right\| .
\end{aligned}
$$

Using the restrictions (a) and (b), we obtain

$$
\lim _{n \rightarrow \infty}\left\|A_{1} z_{n}-A_{1} x^{*}\right\|=0
$$

It follows from (2.12) that

$$
\left\|x_{n+1}-x^{*}\right\|^{2} \leq \alpha_{n}\left\|u-x^{*}\right\|^{2}+\beta_{n}\left\|x_{n}-x^{*}\right\|^{2}+\gamma_{n}\left\|z_{n}-x^{*}\right\|^{2}
$$

Since

$$
\begin{aligned}
\left\|z_{n}-x^{*}\right\|^{2} & =\left\|J_{r_{n}}\left(u_{n}-s_{n} A_{2} u_{n}\right)-x^{*}\right\|^{2} \\
& \leq\left\|\left(I-s_{n} A_{2}\right) u_{n}-\left(I-s_{n} A_{2}\right) x^{*}\right\|^{2} \\
& =\left\|u_{n}-x^{*}\right\|^{2}-2 s_{n}\left(u_{n}-x^{*}, A_{2} u_{n}-A_{2} x^{*}\right\rangle+s_{n}^{2}\left\|A_{2} u_{n}-A_{2} x^{*}\right\|^{2} \\
& \leq\left\|u_{n}-x^{*}\right\|^{2}-2 s_{n} \delta_{2}\left\|A_{2} u_{n}-A_{2} x^{*}\right\|^{2}+s_{n}^{2}\left\|A_{2} u_{n}-A_{2} x^{*}\right\|^{2} \\
& =\left\|u_{n}-x^{*}\right\|^{2}+s_{n}\left(s_{n}-2 \delta_{2}\right)\left\|A_{2} u_{n}-A_{2} x^{*}\right\|^{2},
\end{aligned}
$$

we have

$$
\left\|x_{n+1}-x^{*}\right\|^{2} \leq \alpha_{n}\left\|f\left(x_{n}\right)-x^{*}\right\|^{2}+\left\|x_{n}-x^{*}\right\|^{2}+\gamma_{n} s_{n}\left(s_{n}-2 \delta_{2}\right)\left\|A_{2} u_{n}-A_{2} x^{*}\right\|^{2},
$$


which implies that

$$
\begin{aligned}
& \gamma_{n} s_{n}\left(2 \delta_{2}-s_{n}\right)\left\|A_{2} u_{n}-A_{2} x^{*}\right\|^{2} \\
& \quad \leq \alpha_{n}\left\|f\left(x_{n}\right)-x^{*}\right\|^{2}+\left(\left\|x_{n}-x^{*}\right\|+\left\|x_{n+1}-x^{*}\right\|\right)\left\|x_{n}-x_{n+1}\right\| .
\end{aligned}
$$

Using the restrictions (a) and (b), we obtain

$$
\lim _{n \rightarrow \infty}\left\|A_{2} u_{n}-A_{2} x^{*}\right\|=0 .
$$

Note that

$$
\begin{aligned}
\left\|x_{n+1}-x^{*}\right\|^{2} \leq & \alpha_{n}\left\|u-x^{*}\right\|^{2}+\beta_{n}\left\|x_{n}-x^{*}\right\|^{2}+\gamma_{n}\left\|x_{n}-x^{*}-r_{n}\left(A_{3} x_{n}-A_{3} x^{*}\right)\right\|^{2} \\
\leq & \alpha_{n}\left\|u-x^{*}\right\|^{2}+\beta_{n}\left\|x_{n}-x^{*}\right\|^{2}+\gamma_{n}\left(\left\|x_{n}-x^{*}\right\|^{2}\right. \\
& \left.+\lambda_{n}^{2}\left\|A_{3} x_{n}-A_{3} x^{*}\right\|^{2}-2 \lambda_{n}\left(A_{3} x_{n}-A_{3} x^{*}, x_{n}-x^{*}\right\rangle\right) \\
\leq & \alpha_{n}\left\|u-x^{*}\right\|^{2}+\beta_{n}\left\|x_{n}-x^{*}\right\|^{2}+\gamma_{n}\left\|x_{n}-x^{*}\right\|^{2} \\
& \quad-\lambda_{n} \gamma_{n}\left(2 \delta_{3}-\lambda_{n}\right)\left\|A_{3} x_{n}-A_{3} x^{*}\right\|^{2} .
\end{aligned}
$$

This implies that

$$
\begin{aligned}
& \lambda_{n} \gamma_{n}\left(2 \delta_{3}-\lambda_{n}\right)\left\|A_{3} x_{n}-A_{3} x^{*}\right\|^{2} \\
& \quad \leq \alpha_{n}\left\|u-x^{*}\right\|^{2}+\left(\left\|x_{n}-x^{*}\right\|+\left\|x_{n+1}-x^{*}\right\|\right)\left\|x_{n}-x_{n+1}\right\| .
\end{aligned}
$$

Using the restrictions (a) and (b), we see that

$$
\lim _{n \rightarrow \infty}\left\|A_{3} x_{n}-A_{3} x^{*}\right\|=0 .
$$

Since $T_{\lambda_{n}}$ is firmly nonexpansive, we find that

$$
\begin{aligned}
\left\|u_{n}-x^{*}\right\|^{2} \leq & \left\langle\left(I-\lambda_{n} A_{3}\right) x_{n}-\left(I-\lambda_{n} A_{3}\right) x^{*}, u_{n}-x^{*}\right\rangle \\
\leq & \frac{1}{2}\left(\left\|x_{n}-x^{*}\right\|^{2}+\left\|u_{n}-x^{*}\right\|^{2}-\left\|x_{n}-u_{n}\right\|^{2}-\lambda_{n}^{2}\left\|A_{3} x_{n}-A_{3} x^{*}\right\|^{2}\right. \\
& \left.+2 \lambda_{n}\left(A_{3} x_{n}-A_{3} x^{*}, x_{n}-u_{n}\right\rangle\right) .
\end{aligned}
$$

This in turn implies that

$$
\begin{aligned}
\gamma_{n}\left\|x_{n}-u_{n}\right\|^{2} \leq & \alpha_{n}\left\|f\left(x_{n}\right)-x^{*}\right\|^{2}+\left(\left\|x_{n}-x^{*}\right\|+\left\|x_{n+1}-x^{*}\right\|\right)\left\|x_{n}-x_{n+1}\right\| \\
& +2 \lambda_{n}\left\|A_{3} x_{n}-A_{3} x^{*}\right\|\left\|x_{n}-u_{n}\right\| .
\end{aligned}
$$

Using the restrictions (a) and (b), we see that

$$
\lim _{n \rightarrow \infty}\left\|x_{n}-u_{n}\right\|=0 .
$$


Since $J_{s_{n}}$ is also firmly nonexpansive mapping, we see that

$$
\begin{aligned}
\left\|z_{n}-x^{*}\right\|^{2} \leq & \left\langle\left(I-s_{n} A_{2}\right) u_{n}-\left(I-s_{n} A_{2}\right) x^{*}, z_{n}-x^{*}\right\rangle \\
\leq & \frac{1}{2}\left(\left\|u_{n}-x^{*}\right\|^{2}+\left\|z_{n}-x^{*}\right\|^{2}-\left\|u_{n}-z_{n}-s_{n}\left(A_{2} u_{n}-A_{2} x^{*}\right)\right\|^{2}\right) \\
\leq & \frac{1}{2}\left(\left\|u_{n}-x^{*}\right\|^{2}+\left\|z_{n}-x^{*}\right\|^{2}-\left\|u_{n}-z_{n}\right\|^{2}\right. \\
& \left.+2 s_{n}\left\|u_{n}-z_{n}\right\|\left\|A_{2} u_{n}-A_{2} x^{*}\right\|-s_{n}^{2}\left\|A_{2} u_{n}-A_{2} x^{*}\right\|^{2}\right)
\end{aligned}
$$

which implies that

$$
\left\|z_{n}-x^{*}\right\|^{2} \leq\left\|x_{n}-x^{*}\right\|^{2}-\left\|u_{n}-z_{n}\right\|^{2}+2 s_{n}\left\|u_{n}-z_{n}\right\|\left\|A_{2} u_{n}-A_{2} x^{*}\right\| .
$$

It follows that

$$
\begin{aligned}
\gamma_{n}\left\|u_{n}-z_{n}\right\|^{2} \leq & \alpha_{n}\left\|f\left(x_{n}\right)-x^{*}\right\|^{2}+\left(\left\|x_{n}-x^{*}\right\|+\left\|x_{n+1}-x^{*}\right\|\right)\left\|x_{n}-x_{n+1}\right\| \\
& +2 s_{n}\left\|u_{n}-z_{n}\right\|\left\|A_{2} u_{n}-A_{2} x^{*}\right\| .
\end{aligned}
$$

Using the restrictions (a) and (b), we obtain

$$
\lim _{n \rightarrow \infty}\left\|u_{n}-z_{n}\right\|=0
$$

and

$$
\lim _{n \rightarrow \infty}\left\|y_{n}-z_{n}\right\|=0
$$

Note that

$$
\left(1-\beta_{n}\right)\left\|W_{n} y_{n}-x_{n}\right\| \leq\left\|x_{n}-x_{n+1}\right\|+\alpha_{n}\left\|u-W_{n} y_{n}\right\| .
$$

Using the restrictions (b) and (c), we obtain

$$
\lim _{n \rightarrow \infty}\left\|W_{n} y_{n}-x_{n}\right\|=0
$$

On the other hand, one has

$$
\left\|W_{n} y_{n}-y_{n}\right\| \leq\left\|y_{n}-z_{n}\right\|+\left\|z_{n}-u_{n}\right\|+\left\|u_{n}-x_{n}\right\|+\left\|x_{n}-W_{n} y_{n}\right\| .
$$

Using (2.6), (2.7), (2.8), and (2.9), we find that

$$
\lim _{n \rightarrow \infty}\left\|W_{n} y_{n}-y_{n}\right\|=0
$$

Next, we prove that

$$
\limsup _{n \rightarrow \infty}\left\langle u-\bar{x}, x_{n}-\bar{x}\right\rangle \leq 0
$$


To see this, we choose a subsequence $\left\{x_{n_{i}}\right\}$ of $\left\{x_{n}\right\}$ such that

$$
\limsup _{n \rightarrow \infty}\left\langle u-\bar{x}, x_{n}-\bar{x}\right\rangle=\lim _{i \rightarrow \infty}\left\langle u-\bar{x}, x_{n_{i}}-\bar{x}\right\rangle .
$$

Since $\left\{x_{n_{i}}\right\}$ is bounded, there exists a subsequence $\left\{x_{n_{i}}\right\}$ of $\left\{x_{n_{i}}\right\}$ which converges weakly to $w$. Without loss of generality, we may assume that $x_{n_{i}} \rightarrow q$. Therefore, we see that $y_{n_{i}} \rightarrow q$. We also have $z_{n_{i}} \rightarrow q$.

Next, we show that $q \in \bigcap_{i=1}^{\infty} F\left(S_{i}\right)$. Suppose the contrary, $q \notin C F P S$, i.e., $W q \neq q$. Since $y_{n_{i}} \rightarrow q$, we see from Opial's condition that

$$
\begin{aligned}
\liminf _{i \rightarrow \infty}\left\|y_{n_{i}}-q\right\| & <\liminf _{i \rightarrow \infty}\left\|y_{n_{i}}-W q\right\| \\
& \leq \liminf _{i \rightarrow \infty}\left\{\left\|y_{n_{i}}-W y_{n_{i}}\right\|+\left\|W y_{n_{i}}-W q\right\|\right\} \\
& \leq \liminf _{i \rightarrow \infty}\left\{\left\|y_{n_{i}}-W y_{n_{i}}\right\|+\left\|y_{n_{i}}-q\right\|\right\} .
\end{aligned}
$$

On the other hand, we have

$$
\begin{aligned}
\left\|W y_{n}-y_{n}\right\| & \leq\left\|W y_{n}-W_{n} y_{n}\right\|+\left\|W_{n} y_{n}-y_{n}\right\| \\
& \leq \sup _{x \in K}\left\|W x-W_{n} x\right\|+\left\|W_{n} y_{n}-y_{n}\right\| .
\end{aligned}
$$

In view of Lemma 1.4, we obtain that $\lim _{n \rightarrow \infty}\left\|W y_{n}-y_{n}\right\|=0$. This implies from (2.22) that $\liminf _{i \rightarrow \infty}\left\|y_{n_{i}}-q\right\|<\liminf _{i \rightarrow \infty}\left\|y_{n_{i}}-q\right\|$. This is a contradiction. Thus, we have $q \in$ $\bigcap_{i=1}^{\infty} F\left(S_{i}\right)$.

Now, we are in a position to prove that $q \in\left(A_{1}+M_{1}\right)^{-1}(0)$. Notice that $\frac{z_{n}-y_{n}}{r_{n}}-A_{1} z_{n} \in$ $M_{1} y_{n}$. Let $\mu \in M_{1} v$. Since $M_{1}$ is monotone, we find that

$$
\left\langle\frac{z_{n}-y_{n}}{r_{n}}-A_{1} z_{n}-\mu, y_{n}-v\right\rangle \geq 0
$$

This implies that $\left\langle-A_{1} q-\mu, q-v\right\rangle \geq 0$. This implies that $-A_{1} q \in M_{1} q$, that is, $q \in\left(A_{1}+\right.$ $\left.M_{1}\right)^{-1}(0)$.

Now, we prove that $q \in\left(A_{2}+M_{2}\right)^{-1}(0)$. Notice that $\frac{u_{n}-z_{n}}{s_{n}}-A_{2} u_{n} \in M_{2} z_{n}$. Let $\mu^{\prime} \in M_{2} v^{\prime}$. Since $M_{2}$ is monotone, we find that

$$
\left\langle\frac{u_{n}-z_{n}}{s_{n}}-A_{2} u_{n}-\mu^{\prime}, z_{n}-v^{\prime}\right\rangle \geq 0
$$

This implies that $\left\langle-A_{2} q-\mu^{\prime}, q-v^{\prime}\right\rangle \geq 0$. This implies that $-A_{2} q \in M_{2} q$, that is, $q \in\left(A_{2}+\right.$ $\left.M_{2}\right)^{-1}(0)$.

Next, we show that $q \in E P\left(F, A_{3}\right)$. Since $u_{n}=T_{\lambda_{n}}\left(I-\lambda_{n} A_{3}\right) x_{n}$, for any $y \in C$, we have

$$
F\left(u_{n}, y\right)+\left\langle A_{3} x_{n}, y-u_{n}\right\rangle+\frac{1}{\lambda_{n}}\left\langle y-u_{n}, u_{n}-x_{n}\right\rangle \geq 0
$$

Replacing $n$ by $n_{i}$, we find from (A2) that

$$
\left\langle A_{3} x_{n_{i}}, y-u_{n_{i}}\right\rangle+\left\langle y-u_{n_{i}}, \frac{u_{n_{i}}-x_{n_{i}}}{\lambda_{n_{i}}}\right\rangle \geq F\left(y, u_{n_{i}}\right), \quad \forall y \in C .
$$


Putting $y_{t}=t y+(1-t) q$ for any $t \in(0,1]$ and $y \in C$, we see that $y_{t} \in C$. It follows that

$$
\begin{aligned}
& \left\langle y_{t}-u_{n_{i}}, A_{3} y_{t}\right\rangle \\
& \geq\left\langle y_{t}-u_{n_{i}}, A_{3} y_{t}\right\rangle-\left\langle A_{3} x_{n_{i}}, y_{t}-u_{n_{i}}\right\rangle-\left\langle y_{t}-u_{n_{i}}, \frac{u_{n_{i}}-x_{n_{i}}}{\lambda_{n_{i}}}\right\rangle+F\left(y_{t}, u_{n_{i}}\right) \\
& =\left\langle y_{t}-u_{n_{i}}, A_{3} y_{t}-A_{3} u_{n_{i}}\right\rangle+\left\langle y_{t}-u_{n_{i}}, A_{3} u_{n_{i}}-A_{3} x_{n_{i}}\right\rangle-\left\langle y_{t}-u_{n_{i}}, \frac{u_{n_{i}}-x_{n_{i}}}{\lambda_{n_{i}}}\right\rangle \\
& \quad+F\left(y_{t}, u_{n_{i}}\right) .
\end{aligned}
$$

In view of the monotonicity of $A_{3}$, and the restriction (a), we obtain from (A4) that

$$
\left\langle y_{t}-q, A_{3} y_{t}\right\rangle \geq F\left(y_{t}, q\right)
$$

From (A1) and (A4), we see that

$$
\begin{aligned}
0 & =F\left(y_{t}, y_{t}\right) \leq t F\left(y_{t}, y\right)+(1-t) F\left(y_{t}, q\right) \\
& \leq t F\left(y_{t}, y\right)+(1-t)\left\langle y_{t}-q, A_{3} y_{t}\right\rangle \\
& =t F\left(y_{t}, y\right)+(1-t) t\left\langle y-q, A_{3} y_{t}\right\rangle
\end{aligned}
$$

It follows that

$$
0 \leq F\left(y_{t}, y\right)+(1-t)\left\langle y-w, A_{3} y_{t}\right\rangle, \quad \forall y \in C
$$

It follows from (A3) that $q \in E P\left(F, A_{3}\right)$. Hence,

$$
\limsup _{n \rightarrow \infty}\left\langle u-\bar{x}, x_{n}-\bar{x}\right\rangle \leq 0
$$

Finally, we show that $x_{n} \rightarrow \bar{x}$. Note that

$$
\begin{aligned}
& \left\|x_{n+1}-\bar{x}\right\|^{2} \\
& \quad \leq \alpha_{n}\left\langle u-\bar{x}, x_{n+1}-\bar{x}\right\rangle+\beta_{n}\left\|x_{n}-\bar{x}\right\|\left\|x_{n+1}-\bar{x}\right\|+\gamma_{n}\left\|y_{n}-\bar{x}\right\|\left\|x_{n+1}-\bar{x}\right\| \\
& \quad \leq \alpha_{n}\left\langle u-\bar{x}, x_{n+1}-\bar{x}\right\rangle+\frac{1-\alpha_{n}}{2}\left(\left\|x_{n}-\bar{x}\right\|^{2}+\left\|x_{n+1}-\bar{x}\right\|^{2}\right) .
\end{aligned}
$$

This implies that

$$
\left\|x_{n+1}-\bar{x}\right\|^{2} \leq 2 \alpha_{n}\left\langle u-\bar{x}, x_{n+1}-\bar{x}\right\rangle+\left(1-\alpha_{n}\right)\left\|x_{n}-\bar{x}\right\|^{2} .
$$

Using Lemma 1.1, we find that $\lim _{n \rightarrow \infty}\left\|x_{n}-\bar{x}\right\|=0$. This completes the proof.

\section{Applications}

In this section, we consider some applications of the main results.

Recall that the classical variational inequality is to find an $x \in C$ such that

$$
\langle A x, y-x\rangle \geq 0, \quad \forall y \in C
$$


In this paper, we use $V I(C, A)$ to denote the solution set of the inequality. It is well known that $x \in C$ is a solution of the inequality iff $x$ is a fixed point of the mapping $P_{C}(I-r A)$, where $r>0$ is a constant, $I$ stands for the identity mapping. If $A$ is $\alpha$-inverse-strongly monotone and $r \in(0,2 \alpha]$, then the mapping $I-r A$ is nonexpansive. It follows that $V I(C, A)$ is closed and convex.

Let $g: H \rightarrow(-\infty,+\infty]$ be a proper convex lower semicontinuous function. Then the subdifferential $\partial g$ of $g$ is defined as follows:

$$
\partial f g(x)=\{y \in H: g(z) \geq g(x)+\langle z-x, y\rangle, z \in H\}, \quad \forall x \in H
$$

From Rockafellar [39], we know that $\partial g$ is maximal monotone. It is not hard to verify that $0 \in \partial g(x)$ if and only if $g(x)=\min _{y \in H} g(y)$.

Let $I_{C}$ be the indicator function of $C$, i.e.,

$$
I_{C}(x)= \begin{cases}0, & x \in C \\ +\infty, & x \notin C\end{cases}
$$

Since $I_{C}$ is a proper lower semicontinuous convex function on $H$, we see that the subdifferential $\partial I_{C}$ of $I_{C}$ is a maximal monotone operator. It is clearly that $J_{r} x=P_{C} x, \forall x \in H$, $\left(A_{1}+\partial I_{C}\right)^{-1}(0)=V I\left(C, A_{1}\right)$ and $\left(A_{2}+\partial I_{C}\right)^{-1}(0)=V I\left(C, A_{2}\right)$.

Theorem 3.1 Let $C$ be a nonempty closed convex subset of a Hilbert space $H$ and $F$ a bifunction from $C \times C$ to $\mathbb{R}$ which satisfies (A1)-(A4). Let $A_{1}: C \rightarrow H$ be a $\delta_{1}$-inverse-strongly monotone mapping, $A_{2}: C \rightarrow H$ be a $\delta_{2}$-inverse-strongly monotone mapping, $A_{3}: C \rightarrow H$ be a $\delta_{3}$-inverse-strongly monotone mapping, and $\left\{S_{i}: C \rightarrow C\right\}$ be a family of infinitely nonexpansive mappings. Assume that $\Omega:=\bigcap_{i=1}^{\infty} F\left(S_{i}\right) \cap E P\left(F, A_{3}\right) \cap V I\left(C, A_{1}\right) \cap \operatorname{VI}\left(C, A_{2}\right) \neq \emptyset$. Let $x_{1} \in C$ and $\left\{x_{n}\right\}$ be a sequence generated by

$$
\left\{\begin{array}{l}
z_{n}=P_{C}\left(u_{n}-s_{n} A_{2} u_{n}\right), \\
x_{n+1}=\alpha_{n} f\left(x_{n}\right)+\beta_{n} x_{n}+\gamma_{n} W_{n} P_{C}\left(z_{n}-r_{n} A_{1} z_{n}\right), \quad \forall n \geq 1,
\end{array}\right.
$$

where $u_{n}$ is such that

$$
F\left(u_{n}, y\right)+\left\langle A_{3} x_{n}, y-u_{n}\right\rangle+\frac{1}{\lambda_{n}}\left\langle y-u_{n}, u_{n}-x_{n}\right\rangle \geq 0, \quad \forall y \in C,
$$

$\left\{W_{n}: C \rightarrow C\right\}$ is the sequence generated in (1.2), $\left\{\alpha_{n}\right\},\left\{\beta_{n}\right\}$, and $\left\{\gamma_{n}\right\}$ are sequences in $(0,1)$ such that $\alpha_{n}+\beta_{n}+\gamma_{n}=1$ for each $n \geq 1$ and $\left\{r_{n}\right\},\left\{s_{n}\right\}$, and $\left\{\lambda_{n}\right\}$ are positive number sequences. Assume that the above control sequences satisfy the following restrictions:

(a) $0<a \leq \lambda_{n} \leq b<2 \delta_{3}, 0<a^{\prime} \leq r_{n} \leq b^{\prime}<2 \delta_{1}, 0<\bar{a} \leq s_{n} \leq \bar{b}<2 \delta_{2}$;

(b) $\lim _{n \rightarrow \infty} \alpha_{n}=0$ and $\sum_{n=1}^{\infty} \alpha_{n}=\infty$;

(c) $0<\liminf _{n \rightarrow \infty} \beta_{n} \leq \limsup _{n \rightarrow \infty} \beta_{n}<1$;

(d) $\lim _{n \rightarrow \infty}\left|\lambda_{n}-\lambda_{n+1}\right|=\lim _{n \rightarrow \infty}\left|s_{n}-s_{n+1}\right|=\lim _{n \rightarrow \infty}\left|r_{n}-r_{n+1}\right|=0$.

Then the sequence $\left\{x_{n}\right\}$ converges strongly to $\bar{x}=P_{\Omega} u$. 
Recall that a mapping $T: C \rightarrow C$ is said to be a $k$-strict pseudo-contraction if there exists a constant $k \in[0,1)$ such that

$$
\|T x-T y\|^{2} \leq\|x-y\|^{2}+k\|(I-T) x-(I-T) y\|^{2}, \quad \forall x, y \in C .
$$

Putting $A=I-T$, where $T: C \rightarrow C$ is a $k$-strict pseudo-contraction, we find that $A$ is $\frac{1-k}{2}$-inverse-strongly monotone.

Next, we consider fixed points of strict pseudo-contractions.

Theorem 3.2 Let $C$ be a nonempty closed convex subset of a Hilbert space $H$ and $F$ a bifunction from $C \times C$ to $\mathbb{R}$ which satisfies (A1)-(A4). Let $T_{1}: C \rightarrow H$ be a $k_{1}$-strict pseudocontraction, $T_{2}: C \rightarrow H$ be a $k_{2}$-strict pseudo-contraction, $A_{3}: C \rightarrow H$ be a $\delta$-inversestrongly monotone mapping, and $\left\{S_{i}: C \rightarrow C\right\}$ be a family of infinitely nonexpansive mappings. Assume that $\Omega:=\bigcap_{i=1}^{\infty} F\left(S_{i}\right) \cap E P\left(F, A_{3}\right) \cap F\left(T_{1}\right) \cap F\left(T_{2}\right) \neq \emptyset$. Let $x_{1} \in C$ and $\left\{x_{n}\right\}$ be a sequence generated by

$$
\left\{\begin{array}{l}
z_{n}=\left(1-s_{n}\right) u_{n}+s_{n} T_{2} u_{n}, \\
y_{n}=\left(1-r_{n}\right) u_{n}+r_{n} T_{1} u_{n}, \\
x_{n+1}=\alpha_{n} f\left(x_{n}\right)+\beta_{n} x_{n}+\gamma_{n} W_{n} y_{n}, \quad \forall n \geq 1,
\end{array}\right.
$$

where $u_{n}$ is such that

$$
F\left(u_{n}, y\right)+\left\langle A_{3} x_{n}, y-u_{n}\right\rangle+\frac{1}{\lambda_{n}}\left\langle y-u_{n}, u_{n}-x_{n}\right\rangle \geq 0, \quad \forall y \in C,
$$

$\left\{W_{n}: C \rightarrow C\right\}$ is the sequence generated in (1.2), $\left\{\alpha_{n}\right\},\left\{\beta_{n}\right\}$, and $\left\{\gamma_{n}\right\}$ are sequences in $(0,1)$ such that $\alpha_{n}+\beta_{n}+\gamma_{n}=1$ for each $n \geq 1$ and $\left\{r_{n}\right\},\left\{s_{n}\right\}$, and $\left\{\lambda_{n}\right\}$ are positive number sequences. Assume that the above control sequences satisfy the following restrictions:

(a) $0<a \leq \lambda_{n} \leq b<2 \delta, 0<a^{\prime} \leq r_{n} \leq b^{\prime}<1-k_{1}, 0<\bar{a} \leq s_{n} \leq \bar{b}<1-k_{2}$;

(b) $\lim _{n \rightarrow \infty} \alpha_{n}=0$ and $\sum_{n=1}^{\infty} \alpha_{n}=\infty$;

(c) $0<\liminf _{n \rightarrow \infty} \beta_{n} \leq \limsup _{n \rightarrow \infty} \beta_{n}<1$;

(d) $\lim _{n \rightarrow \infty}\left|\lambda_{n}-\lambda_{n+1}\right|=\lim _{n \rightarrow \infty}\left|s_{n}-s_{n+1}\right|=\lim _{n \rightarrow \infty}\left|r_{n}-r_{n+1}\right|=0$.

Then the sequence $\left\{x_{n}\right\}$ converges strongly to $\bar{x}=P_{\Omega} u$.

Proof Taking $A_{i}=I-T_{i}$, wee see that $A_{i}: C \rightarrow H$ is a $\delta_{i}$-strict pseudo-contraction with $\delta_{i}=\frac{1-k_{i}}{2}$ and $F\left(T_{i}\right)=V I\left(C, A_{i}\right)$ for $i=1,2$. In view of Theorem 3.1, we find the desired conclusion immediately. 


\section{References}

1. Cho, SY, Li, W, Kang, SM: Convergence analysis of an iterative algorithm for monotone operators. J. Inequal. Appl. 2013, $199(2013)$

2. $\mathrm{Wu}, \mathrm{C}$ : Convergence of algorithms for an infinite family nonexpansive mappings and relaxed cocoercive mappings in Hilbert spaces. Adv. Fixed Point Theory 4, 125-139 (2014)

3. Zegeye, $\mathrm{H}$, Shahzad, $\mathrm{N}$ : Strong convergence theorem for a common point of solution of variational inequality and fixed point problem. Adv. Fixed Point Theory 2, 374-397 (2012)

4. Cho, SY, Kang, SM: Approximation of common solutions of variational inequalities via strict pseudocontractions. Acta Math. Sci. 32, 1607-1618 (2012)

5. Cho, SY, Qin, X: On the strong convergence of an iterative process for asymptotically strict pseudocontractions and equilibrium problems. Appl. Math. Comput. 235, 430-438 (2014)

6. Zhang, M: Iterative algorithms for common elements in fixed point sets and zero point sets with applications. Fixed Point Theory Appl. 2012, Article ID 21 (2012)

7. Kim, JK: Strong convergence theorems by hybrid projection methods for equilibrium problems and fixed point problems of the asymptotically quasi- $\phi$-nonexpansive mappings. Fixed Point Theory Appl. 2011, Article ID 10 (2011)

8. Cho, SY, Kang, SM: Approximation of fixed points of pseudocontraction semigroups based on a viscosity iterative process. Appl. Math. Lett. 24, 224-228 (2011)

9. Cho, SY, Qin, X, Kang, SM: Iterative processes for common fixed points of two different families of mappings with applications. J. Glob. Optim. 57, 1429-1446 (2013)

10. Luo, H, Wang, Y: Iterative approximation for the common solutions of a infinite variational inequality system for inverse-strongly accretive mappings. J. Math. Comput. Sci. 2, 1660-1670 (2012)

11. Zhang, Q: Iterative approximation of solutions of monotone quasi-variational inequalities via nonlinear mappings. J. Fixed Point Theory 2014, Article ID 1 (2014)

12. Jeong, JU: Fixed point solutions of generalized mixed equilibrium problems and variational inclusion problems for nonexpansive semigroups. Fixed Point Theory Appl. 2014, Article ID 57 (2014)

13. Cho, SY, Qin, X, Wang, L: Strong convergence of a splitting algorithm for treating monotone operators. Fixed Point Theory Appl. 2014, 94 (2014)

14. Qin, X, Cho, SY: Implicit iterative algorithms for treating strongly continuous semigroups of Lipschitz pseudocontractions. Appl. Math. Lett. 23, 1252-1255 (2010)

15. Chen, JH: Iterations for equilibrium and fixed point problems. J. Nonlinear Funct. Anal. 2013, Article ID 4 (2013)

16. Qing, Y, Cho, SY: Proximal point algorithms for zero points of nonlinear operators. Fixed Point Theory Appl. 2014, 42 (2014)

17. Zhang, M: Strong convergence of a viscosity iterative algorithm in Hilbert spaces. J. Nonlinear Funct. Anal. 2014 Article ID 1 (2014)

18. $\mathrm{Wu}, \mathrm{C}, \mathrm{LV}, \mathrm{S}:$ Bregman projection methods for zeros of monotone operators. J. Fixed Point Theory 2013, Article ID 7 (2013)

19. LV, S, Hao, Y: Some results on continuous pseudo-contractions in a reflexive Banach space. J. Inequal. Appl. 2013, 538 (2013)

20. Qin, X, Cho, YJ, Kang, SM: Convergence theorems of common elements for equilibrium problems and fixed point problems in Banach spaces. J. Comput. Appl. Math. 225, 20-30 (2009)

21. Qin, X, Agarwal, RP: Shrinking projection methods for a pair of asymptotically quasi- $\phi$-nonexpansive mapping. Numer. Funct. Anal. Optim. 31, 1072-1089 (2010)

22. Kim, KS, Kim, JK, Lim, WH: Convergence theorems for common solutions of various problems with nonlinear mapping. J. Inequal. Appl. 2014, 2 (2014)

23. LV, S: Strong convergence of a general iterative algorithm in Hilbert spaces. J. Inequal. Appl. 2013, 19 (2013)

24. Wang, Z, Lou, W: A new iterative algorithm of common solutions to quasi-variational inclusion and fixed point problems. J. Math. Comput. Sci. 3, 57-72 (2013)

25. Qin, $X$, Shang, M, Su, Y: Strong convergence of a general iterative algorithm for equilibrium problems and variational inequality problems. Math. Comput. Model. 48, 1033-1046 (2008)

26. Qin, X, Shang, M, Zhou, H: Strong convergence of a general iterative method for variational inequality problems and fixed point problems in Hilbert spaces. Appl. Math. Comput. 200, 242-253 (2008)

27. Chang, SS, Lee, HWJ, Chan, CK: A new method for solving equilibrium problem fixed point problem and variational inequality problem with application to optimization. Nonlinear Anal. 70, 3307-3319 (2009)

28. Qin, $X, C h o, S Y$, Wang, L: Iterative algorithms with errors for zero points of $m$-accretive operators. Fixed Point Theory Appl. 2013, 148 (2013)

29. Song, J, Chen, M: On generalized asymptotically quasi- $\phi$-nonexpansive mappings and a Ky Fan inequality. Fixed Point Theory Appl. 2013, 237 (2013)

30. Zhang, QN: Common solutions of equilibrium and fixed point problems. J. Inequal. Appl. 2013, 425 (2013)

31. Qin, $X, C h o, S Y$, Wang, L: A regularization method for treating zero points of the sum of two monotone operators. Fixed Point Theory Appl. 2014, 75 (2014)

32. Nadezhkina, N, Takahashi, W: Weak convergence theorem by an extragradient method for nonexpansive mappings and monotone mappings. J. Optim. Theory Appl. 128, 191-201 (2006)

33. Qin, X, Cho, SY, Kang, SM: An extragradient-type method for generalized equilibrium problems involving strictly pseudocontractive mappings. J. Glob. Optim. 49, 679-693 (2011)

34. Liu, LS: Ishikawa and Mann iteration process with errors for nonlinear strongly accretive mappings in Banach spaces. J. Math. Anal. Appl. 194, 114-125 (1995)

35. Blum, E, Oettli, W: From optimization and variational inequalities to equilibrium problems. Math. Stud. 63, 123-145 (1994)

36. Shimoji, K, Takahashi, W: Strong convergence to common fixed points of infinite nonexpansive mappings and applications. Taiwan. J. Math. 5, 387-404 (2001)

37. Cho, SY: Strong convergence of an iterative algorithm for sums of two monotone operators. J. Fixed Point Theory 2013, Article ID 6 (2013) 
38. Suzuki, T: Strong convergence of Krasnoselskii and Mann's type sequences for one-parameter nonexpansive semigroups without Bochne integrals. J. Math. Anal. Appl. 305, 227-239 (2005)

39. Rockafellar, RT: On the maximality of sums of nonlinear monotone operators. Trans. Am. Math. Soc. 149, 75-88 (1970)

\#DIGITAL OBJECT IDENTIFIER

Cite this article as: Sun: Hybrid methods for common solutions in Hilbert spaces with applications. Journal of Inequalities and Applications \#CITATION

Submit your manuscript to a SpringerOpen ${ }^{\circ}$ journal and benefit from:

- Convenient online submission

- Rigorous peer review

- Immediate publication on acceptance

- Open access: articles freely available online

- High visibility within the field

- Retaining the copyright to your article

Submit your next manuscript at $\gg$ springeropen.com 